\title{
CREACIÓN Y MANTENIMIENTO DEL CONOCIMIENTO COMPARTIDO: CONTRIBUCIÓN DE LA UNIVERSITY OF SOUTHAMPTON
}

\author{
Les Carr, Alma Swan y Stevan Harnad
}

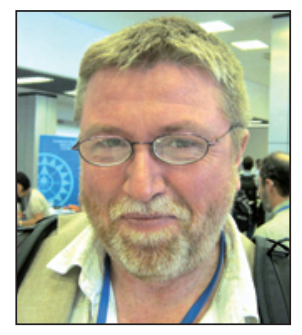

Leslie Carr experimenta desde los 80s con sistemas multimedia, hipertexto, bibliotecas digitales y sistemas de gestión del conocimiento, pero en los últimos años trabaja en temas de acceso abierto y repositorios digitales. Su objetivo es animar a los investigadores y científicos a que se responsabilicen de sus propios activos de propiedad intelectual, y construyan entornos de información del que todos nos beneficiaríamos: repositorios, servicios de análisis, mapas comunitarios, sistemas de seguimiento de ideas.

Es miembro de la iniciativa Web Science Research y co-director del Web Science Doctoral Training Centre, director técnico del equipo que elabora el software EPrints, gerente del repositorio ECS y también escribe un blog sobre su experiencia como RepositoryMan.

Profesor de la University of Southampton, dirige los masters Web technologies y Web science. Da clases sobre fundamentos de la Web, métodos de investigación Web, javascript, metodología de programación, hipertexto y tecnologías web. También es gerente de laboratorios ECS Computer Science Teaching y desarrollador de Animated Code Annotater para la enseñanza de estos cursos.

School of Electronics and Computer Science University of Southampton Highfield, Southampton SO17 1BJ lac@ecs.soton.ac.uk

http://www.ecs.soton.ac.uk/

http://users.ecs.soton.ac.uk/lac/

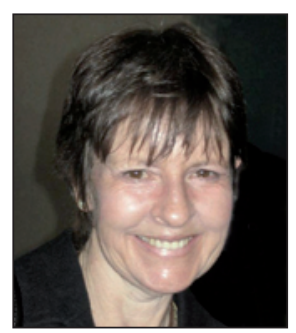

Alma Swan es consultora en el campo de la comunicación científica. Directora de Key Perspectives Ltd., da clases en la School of Electronics \& Computer Science (ECS) de la University of Southampton y la Business School de la University of Warwick. Coordinadora de Enabling Open Scholarship (EOS), la organización global de universidades que promueven los principios de la ciencia abierta en la comunidad académica.

Realiza investigaciones de mercado, modelos de negocio, gestión y evaluación de proyectos, prácticas y comportamientos de la investigación en comunicación, y estudia y promociona nuevas formas de comunicación científica en la era de la Web. Escribe y hace presentaciones frecuentes sobre cuestiones de comunicación científica.

Es licenciada y doctora por la University of Southampton y MBA de la Warwick Business School. Miembro de la Society of Biology (Reino Unido) y bióloga colegiada, es miembro electo de la junta de gobierno de Euroscience (asociación europea para la promoción de la ciencia y la tecnología) y ex editora de su revista online The euroscientist.

Key Perspectives Ltd 48 Old Coach Road, Playing Place Truro TR3 6ET, United Kingdom aswan@keyperspectives.co.uk http://www.keyperspectives.co.uk

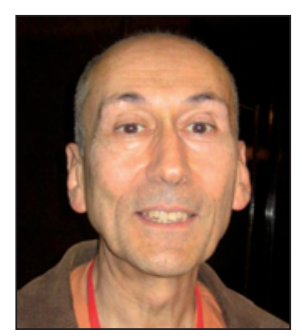

Stevan Harnad nació en Budapest, Hungría. Realizó sus estudios de pregrado en la McGill University, Montréal, y su posgrado en psicología en la Princeton University. Es catedrático de investigación en ciencia cognitiva de la Université du Québec à Montréal (UQAM) y profesor de ciencia cognitiva de la University of Southampton. Miembro externo de la Academia Húngara de Ciencias. Investiga temas de clasificación, comunicación, cognición y concienciación.

Ha escrito sobre percepción de las categorías, símbolos, origen del lenguaje, lateralización, test de Turing, cognición distribuida, cienciometría y concienciación.

Fue fundador (1978) y editor hasta 2002 de Behavioral and brain sciences, revista publicada por Cambridge University Press; de Psycoloquy, revista electrónica patrocinada por la American Psychological Association; de CogPrints, repositorio electrónico de en ciencias cognitivas de la University of Southampton, y del American scientist open access forum (desde 1998). Es un activo promotor del acceso abierto (EPrints, Enabling Open Scholarship (EOS), Open access scholarly information sourcebook (Oasis), y Campus open access policies de Sparc. 


\section{Resumen}

Se presenta un recorrido histórico por las contribuciones que la School of Electronics and Computer Science de la University of Southampton ha realizado para promover el acceso abierto (OA), liderando este movimiento a nivel mundial. Se discuten las ventajas e inconvenientes de las llamadas vía verde (auto-archivo en repositorios) y vía dorada (autor paga). Se razona por qué ha sido un error histórico promover la vía dorada al acceso abierto, en vez de la vía verde, y se tratan las diferentes políticas a seguir para conseguir el OA universal a la ciencia.

\section{Palabras clave}

Acceso abierto, OA, Políticas, Beneficios, Vía dorada, Vía verde, Mandatos, Repositorios, Común cognitivo, Universidad de Southampton.

\section{Title: Creating and curating the cognitive commons: Southampton's contribution}

Historical overview of the significant contributions the School of Electronics and Computer Science at the University of Southampton has made to promote open access (OA), leading this movement worldwide. The advantages and disadvantages of the so-called greenway (self-archiving in repositories) and golden road (author pays) are presented. An analysis is made of the historical mistake of promoting the golden road to open access, instead of the green road. The different policies to achieve universal OA to science are discussed.

\section{Keywords}

Open access, OA, Policies, Benefits, Golden road, Green road, Mandates, Repositories, Cognitive commons, University of Southampton.

Carr, Les; Swan, Alma; Harnad, Stevan. "Creación y mantenimiento del conocimiento compartido: contribución de la University of Southampton". El profesional de la información, 2010, enero-febrero, v. 20, n. 1, pp. 102-110.

\section{Cuatro revoluciones en los medios de producción del conocimiento}

La cognición es pensamiento, y el producto del pensamiento es conocimiento. Nuestra especie tiene una capacidad única, que es el lenguaje (Harnad, 2011), y el hecho de hablar nos faculta para "leer la mente" de los demás y conocer sus pensamientos, creando y gestionando los conocimientos de forma conjunta e interactiva gracias a su compartición (Dror; Harnad, 2009). La primera y más grande revolución cognitiva (Harnad, 1991) -el nacimiento del lenguaje- tuvo lugar hace unos 300.000 años y creó la tradición oral. La segunda revolución fue la escritura hace 6.000 años, y produjo los registros escritos. La imprenta fue la tercera hace 600 años, y dio lugar a los archivos públicos. La cuarta revolución cognitiva está teniendo lugar en nuestra era. Con la invención de internet y la Web $^{9}$ la humanidad está a punto de crear un común cognitivo (Poynder; Harnad, 2007) -un medio de colaboración global en el que el conocimiento puede crearse y comunicarse a la velocidad del pensamiento.

Cuando el lenguaje fue evolucionando, su reciprocidad y uso mutuo fue lo que le dio su poder, lo que aceleró de forma espectacular la codificación permanente de nuestra capacidad de lenguaje en nuestro genoma y en el cerebro: funda- mentalmente el lenguaje es distribuido e interactivo. Nunca habría evolucionado si compartir nuestros pensamientos no hubiera sido beneficioso para nuestra supervivencia y la reproducción de la especie. Por ello el lenguaje ha sido llamado una forma de "altruismo recíproco", practicado porque está en los intereses de nuestros genes egoístas. En su origen los pensamientos eran compartidos libremente -en la familia y dentro de la tribu- en beneficio de todos, y se transmitieron de generación en generación a través de la tradición oral. La escritura hizo posible preservar los pensamientos del hablante de manera independiente (verba volant, scripta manent). Sin embargo fue en gran medida la imprenta la que convirtió los pensamientos -en forma de palabras impresas- en una mercancía que podía comprarse y venderse. La imprenta hizo posible -de hecho fue necesario- solicitar un pago a cambio de las palabras, con el fin de cubrir los considerables costes de la difusión de la palabra escrita.

La posibilidad de cobrar "por los pensamientos" no fue negativo: fue algo natural de la evolución cultural hacia la especialización y la división del trabajo. La compra y venta de productos y servicios evitó que cada uno fuera un maestro de la autosuficiencia teniendo todas las habilidades y mercancías para la supervivencia. Pero hay dos efectos secundarios negativos de la era de la escritura y la impresión: 
el primero fue la poca velocidad (Harnad, 2003). Nuestros cerebros se han adaptado biológicamente de forma específica a hablar (y gesticular): la velocidad del pensamiento es aproximadamente la velocidad del habla, de una forma muy interactiva. La escritura a mano conservó los registros de forma permanente, pero a costa de perder la interactividad del diálogo en tiempo real. La impresión y el correo postal ampliaron el ámbito y el alcance de la palabra escrita, pero el tiempo de respuesta entre que se enviaba un texto, alguien lo leía y contestaba algo estaba aún muy desfasado de la potencial velocidad del pensamiento. Ha sido sólo con la posibilidad del correo electrónico y la interacción instantánea en red -un skywriting distribuido y mundial (Harnad, 1990) - que la cognición ha recuperado su potencial nativo para funcionar a la velocidad del pensamiento para la cual nuestro cerebro está optimizado.

Por lo tanto la era de internet ha superado el primer efecto secundario negativo de la escritura y la era de la impresión: el tiempo de respuesta. Estamos a punto de superar el segundo, que se refiere al acceso al conocimiento. Tanto en la era de los manuscritos iluminados como en la de la imprenta de Gutenberg disfrutar de las ventajas de los nuevos medios escritos tenía precio: la escritura a mano había costado a los escribas un tiempo y esfuerzo considerable, y la producción y distribución de las obras impresas era también un proceso caro que tenía que recuperar sus costos mediante tarifas de acceso para que fuera sostenible. Esta restricción no fue problema para comercializar las publicaciones: los que escribían para ganarse la vida mediante la venta de sus palabras se beneficiaron de los obstáculos de acceso naturales y de los peajes de acceso de la era Gutenberg.

Pero siempre ha habido excepciones especiales (Harnad, 2001). Muchos académicos y la mayoría de los científicos no escriben para vender sus palabras: escriben con el fin de comunicar su conocimiento, informando de los resultados de su investigación para que otros investigadores y científicos puedan acceder, utilizar, aplicar y elaborar nuevas investigaciones a partir de ellos, en la empresa de la investigación científica de reciprocidad, acumulación de conocimientos y de colaboración. Sin embargo, estos autores no comerciales, que escriben para que su investigación tenga impacto y no para obtener ingresos, tienen que aceptar las limitaciones sobre los objetivos, el alcance, la captación de lectores y el impacto de sus resultados que se impusieron necesariamente en todos los autores -tanto comerciales como no- por las limitaciones reales tecnológicas y económicas de la era Gutenberg.

Nuestro medio de comunicación online post-Gutenberg al fin hace posible que los autores no comerciales -los investigadores académicos y científicos- liberen sus escritos de las barreras y de los peajes de acceso que habían existido desde el comienzo de la era de la imprenta.

\section{Acceso abierto}

Open access (OA) significa acceso online gratuito. Lo que hizo posible el acceso abierto fue la llegada del medio online en red: internet y finalmente la Web dieron poder a los autores de obras digitales para ofrecerlas gratis online si así lo deseaban.
El término "acceso abierto" fue acuñado por primera vez por la Budapest Open Access Initiative (BOAI) ${ }^{4}$, un proyecto de difusión de dicha idea patrocinado por el Open Society Institute (OSI) en 2001. Pero la idea y la práctica de ofrecer acceso gratuito online se inició mucho antes de la BOA/ y de la adopción de las siglas "OA". Los inventores de Unix ${ }^{28} y$ de internet ${ }^{9}$-en su mayoría ingenieros informáticos- ya habían puesto en OA sus trabajos de investigación en archivos FTP anonymous 3 por lo menos desde la década de 1970. Con la invención de la Web ${ }^{29}$ en 1990, los sitios web se convirtieron pronto en la manera preferida de auto-archivar documentos. Los físicos de altas energías -que ya habían compartido sistemáticamente sus documentos en papel antes de internet, y luego por correo electrónico cuando fue posible- comenzaron el auto-archivo en $\operatorname{arXiv}^{2}$, un repositorio web centralizado de física, matemáticas e informática, en 1991. Muchas personas de otras disciplinas han seguido el ejemplo de los físicos y de los científicos de la computación.

En 1994 en la University of Southampton se originó una "Propuesta subversiva" (Harnad, 1994, 1995) para que todos los artículos de revistas arbitradas (revisadas por pares) estuvieran en acceso gratuito online para todos. La propuesta también identificó, ya entonces, la manera de cubrir los gastos de publicación si finalmente el autoarchivo en OA hiciera las suscripciones insostenibles: tarifas para publicar los artículos en lugar de tarifas de suscripción para acceder a ellos. Las primeras revistas en OA comenzaron a publicarse en $1989^{13}$, y la mayoría eran versiones electrónicas de revistas de suscripción y revistas subvencionadas sólo online.

Internet ha superado el primer efecto secundario negativo de la escritura y la era de la impresión: el tiempo de respuesta

Mientras tanto la Propuesta subversiva de auto-archivo de 1994 fue en gran parte letra muerta: en la siguiente década, la tasa de auto-archivo por parte de los autores se situó sólo en torno a un $15-20 \%$ de la producción anual de artículos evaluados (Björk, 2010). Y la proporción de artículos publicados en revistas de acceso abierto fue aún menor (Gargouri; Harnad, 2010). Los archivos centralizados similares a arXiv de otras disciplinas (por ejemplo, CogPrints ${ }^{6}$ para las ciencias cognitivas, creado y alojado en la University of Southampton en 1997) fallaron en aumentar la tasa de auto-archivo en OA. Tampoco funcionó la creación del American Scientist Open Access Forum ${ }^{1}$ en 1998 -mantenido por la Sigma Xi Scientific Research Society ${ }^{22}$ y alojado en la University of Southampton.

En 1999 la Open Archives Initiative (OAI) ${ }^{14}$ desarrolló un protocolo de etiquetado de metadatos con el propósito de hacer todos los archivos abiertos "interoperables". Esto significaba que depositar un documento a nivel local en cualquier archivo institucional compatible con el protocolo $O A$ I sería equivalente a depositarlo en un archivo abierto central global, consultable en todo su conjunto de forma transparente. En 2000 en la University of Southampton se elaboró el software libre EPrints ${ }^{8}$ (adaptando el software CogPrints para que fuera compatible con el protocolo OAl y también 


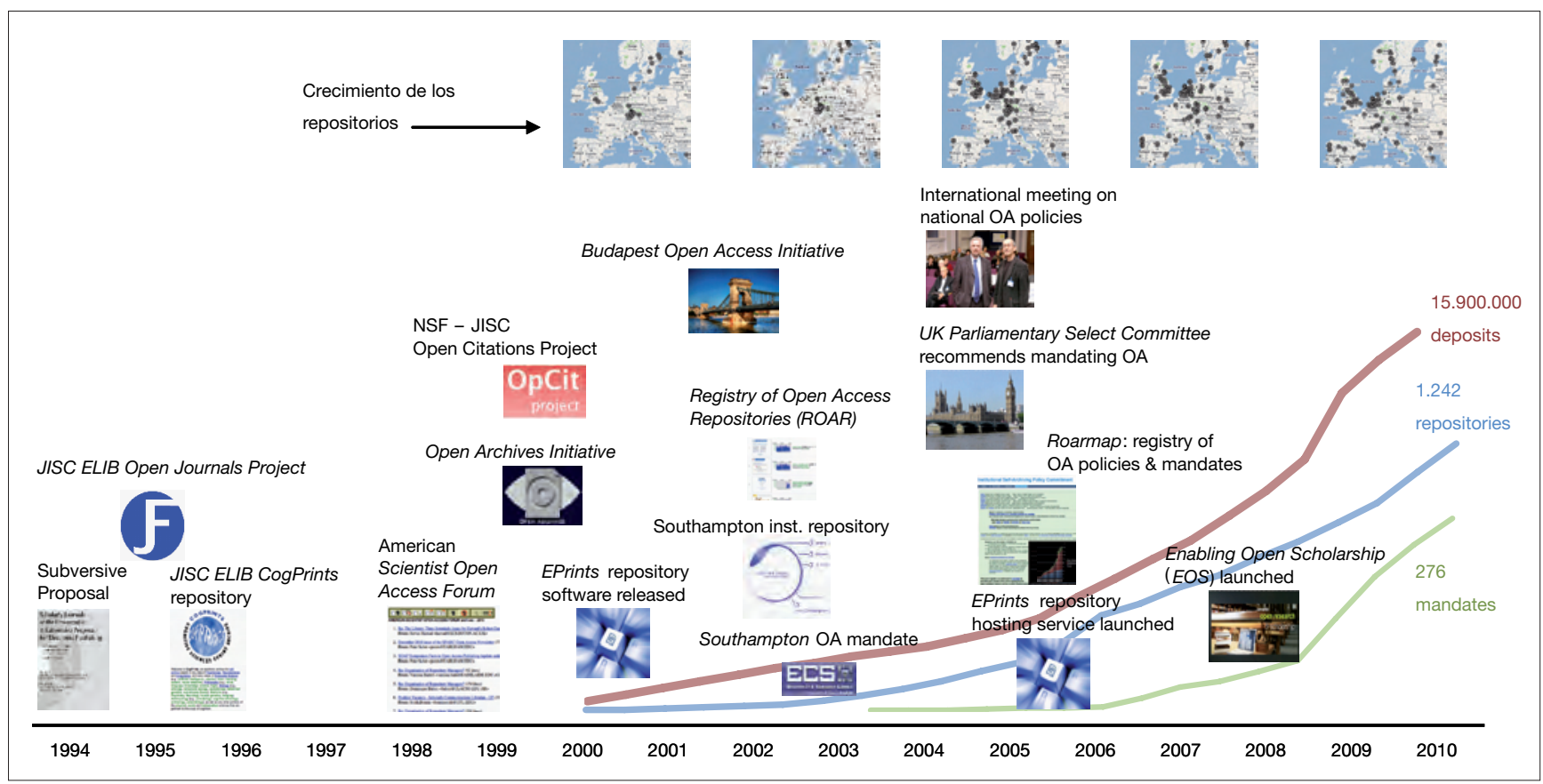

El gráfico ilustra cómo una serie de hitos de Southampton han contribuido al crecimiento del open access desde la publicación de la Propuesta subversiva de 1994, la creación del software Eprints en el año 2000, y la adopción y promoción de los mandatos a partir del mandato de la ECS Southampton en 2002.

para hacerlo genérico, instalable en cualquier máquina). Así fue posible que todas las universidades crearan sus propios archivos abiertos compatibles con OAl, los llamados "repositorios institucionales" (RI). A partir de ahí se fueron creando muchos RIs en todo el mundo, siendo su crecimiento monitorizado desde 2001 por el Registry of OA Repositories $(R O A R)^{17}$ de la University of Southampton. Sin embargo la mayoría de Rls se han mantenido vacíos porque el $85 \%$ de los investigadores todavía no auto-archivan sus trabajos.

Lawrence (2001) publicó un artículo en Nature dando cuenta de que los artículos de informática en OA se citan más que los que no están. Muchos estudios de seguimiento posteriores -recogidos en una bibliografía cada vez mayor titulada "The effect of open access and downloads ('hits') on citation impact", creada y alojada en la University of Southampton (Hitchcock, 2011)- han confirmado que esta "ventaja OA del impacto" también se produce en otros campos académicos y científicos. Pero incluso esta ventaja del OA no ha sido suficiente para convencer e inducir al $85 \%$ de los autores que no auto-archivan a hacerlo.

\section{Mandatos de acceso abierto}

En 1998 el American Scientist Open Access Forum ya había propuesto que las universidades y quienes financian la investigación dictaran un mandato de auto-archivo (es decir, que sea un requisito, como una extensión natural de la obligación de publicar o perecer). Después de haberlo propuesto por primera vez, la School of Electronics and Computer Science de la University of Southampton también se convirtió en la primera del mundo en adoptar un mandato de auto-archivo en OA en 2002, y lo ofreció como modelo a otras instituciones en el OSI Eprints handbook ${ }^{16}$ en 2003.

La University of Southampton ha proporcionado un Registry of open access materials archiving policies (Roarmap) ${ }^{18}$ para el registro y seguimiento del crecimiento de los mandatos de OA desde 2003. El primer mandato OA para toda una universidad fue aprobado por la Queensland University of Technology, de Australia, y el primer mandato europeo fue el de la Universidade do Minho, de Portugal, ambos en 2004.

Asimismo, en 2004 el Parliamentary Select Committee on Science and Technology ${ }^{24}$ del Reino Unido recomendó que las universidades y quienes financian la investigación promulgaran un mandato OA -de nuevo a instancias de la University of Southampton. En el mismo año, el House Appropriations Committee ${ }^{25}$ de los EUA recomendó también que los National Institutes of Health $(\mathrm{NIH})$ ordenaran el OA.

En 2005 el Wellcome Trust se convirtió en el primer financiador de investigación mundial con mandato de OA. Y en el mismo año, los NIH aprobaron la solicitud de OA en lugar de mandato. La simple "solicitud" fracasó y en 2007 obligó a depositar los resultados de la investigación por mandato. El gobierno británico no siguió la citada recomendación del Committee, pero en pocos años los siete Research Councils ${ }^{26}$ del Reino Unido adoptaron un mandato de autoarchivo cada uno por su cuenta.

\section{http://www.wellcome.ac.uk/node3302.html http://publicaccess.nih.gov/policy.htm}

Un incentivo más para promulgar mandatos de OA fue que los resultados del Research Assessment Exercise (RAE) del Reino Unido -sistema en el que los colegas pares revisan y clasifican los artículos de investigación de todos los departamentos de las universidades del Reino Unido cada seis años- estuvieron muy correlacionados con los indicadores de citas que el OA ha demostrado aumentar (Harnad; Carr; Brody; Oppenheim, 2003; Harnad; Carr; Gingras, 2008). La University of Southampton ha dado un gran impulso al desarrollo de métricas del OA para realizar un seguimiento, evaluar y poder premiar el uso de la investigación y su impacto. Crearon Citebase 5 como un modelo de motor cienciométrico para evaluar la investigación y navegar por las citas, 
y con IRStats ${ }^{10}$ recopilan datos del uso de los repositorios institucionales.

Dos estudios internacionales de autores multidisciplinares (Swan, 2005) informaron de un hallazgo estratégico, fundamental y práctico acerca de por qué el crecimiento del OA había sido tan lento: aunque la mayoría de autores no auto-archivan, más del $90 \%$ de ellos indicaron que autoarchivarían si su donantes o sus instituciones se lo mandaran -y más del $80 \%$ de ellos indicó que lo haría de buena gana. Los estudios de Sale (2006a y 2006b) en Australia han confirmado que a partir de los dos años de la adopción de un mandato, las tasas de cumplimiento sobrepasan el $60 \%$ yendo claramente en camino del $100 \%$. Según Roarmap ${ }^{18}$ el número de mandatos se acerca ya a 200 en todo el mundo, entre ellos Harvard, MIT, ETH Zurich y University College London, así como el European Research Council y la Comisión Europea.

\section{Acceso abierto verde y dorado}

El auto-archivo se ha dado en llamar "vía verde" al acceso abierto, para distinguirlo de la publicación de revistas directamente en acceso abierto (autor paga), que se llama la "vía dorada" (Harnad; Brody; Vallières et al., 2004). El error más frecuente sobre el OA es pensar que OA sólo significa vía dorada (el de las editoriales). De hecho, el camino más rápido y más seguro es el verde, el auto-archivo (Harnad, 2007), por dos razones fundamentales: (1) proporcionar OA verde está enteramente en las manos (e intereses) de los proveedores de la propia investigación, la comunidad mundial de investigación; y (2) el OA verde puede obtenerse por mandato, mientras que la vía dorada depende de la comunidad editorial y no puede ser obligada.

Ha sido un gran error estratégico fomentar la vía dorada. Antes hay que promover la vía verde, ya que está en manos de los autores

Por lo tanto la vía verde tiene que venir primero, y debe ser universalmente obligatoria, por las universidades y por las instituciones financiadoras. Ha sido un gran error estratégico esperar la vía dorada ${ }^{21}$. Si a pesar de los beneficios del OA la mayoría de los autores no apoyan la vía verde espontáneamente por su propia voluntad, sin coste alguno, y sin tener que abandonar la revista de su elección para publicar, entonces ciertamente no apoyarán la vía dorada, que tiene un coste adicional, y tienen que publicar en una revista $\mathrm{OA}$ en lugar de en la revista preferida. Además las instituciones no tienen dinero para pagar a sus autores los gastos de publicación en abierto, pues el dinero sigue teniéndose que dedicar al pago de suscripciones. Tampoco pueden cancelarse las suscripciones a revistas mientras su contenido no sea accesible de otra forma que la que abona la institución. Por otra parte, el precio de la publicación en vía dorada sigue siendo mucho mayor de lo que debería ser.

Si primero se promulgaran mandatos universales, entonces, siempre que el resultado de la vía verde universal hiciera inviable a los editores cubrir los costes de publicación mediante suscripciones (porque las instituciones cancelarían sus suscripciones a revistas), el efecto natural sería inducir a los editores de revistas a recortar costos, reduciendo su tamaño, y pasando a la vía dorada. Entonces el ahorro anual producido por las cancelaciones institucionales serviría para pagar los costos de publicación de los autores institucionales en la vía dorada. Los costos por artículo, sin embargo, serían considerablemente más bajos después de que la vía verde universal hubiera hecho insostenibles las suscripciones, porque las revistas ya no necesitarían proporcionar edición impresa y online. Todo el acceso y el archivo se habrá encomendado a la red distribuida de repositorios institucionales. Las revistas sólo proporcionarán el servicio de revisión por pares y la maquetación (Harnad, 2010a), y el ahorro institucional será más que suficiente para cubrir sus precios (Harnad, 2009).

Si en lugar de ordenar primero la vía verde y esperar a que los mandatos se propaguen a nivel mundial y hagan sus efectos naturales, las instituciones dedican parte de sus escasos fondos a pagar de forma preventiva la alta tarifa de la vía dorada, recibirán muy poco OA a cambio de su dinero y se reforzará la publicación en vía dorada al precio y al modus operandi actuales, sin lograr ver la vía verde universal que está ya casi a su alcance. Los compromisos de los consorcios institucionales (como Scoap ${ }^{20}$ ) -destinados a negociar los precios de las revistas a cambio de su conversión a la vía dorada- no son sostenibles, porque, a diferencia de las suscripciones, se pueden cancelar en cualquier momento por parte de las instituciones individuales sin perder el acceso (porque las revistas se han convertido a la vía dorada).

Por lo tanto la única forma escalable, sostenible y segura de alcanzar el OA universal es el mandato de la vía verde en primer lugar, y convertirse a la vía dorada sólo cuando la vía verde universal haya hecho las suscripciones insostenibles. Eso significa que las cancelaciones institucionales forzarán a los editores a reducir su función sólo a la prestación del servicio de revisión por pares, mientras que al mismo tiempo se habrán liberado los fondos de las suscripciones para pagar por ello.

Hay que esperar a promocionar la vía dorada hasta que la vía verde universal haya hecho las suscripciones insostenibles

Este escenario fue confirmado de forma independiente por el Informe Houghton (Houghton et al., 2009), un análisis económico que se centró en los costos de publicación. Concluye que la publicación universal en vía dorada podría eventualmente ahorrar dinero a las instituciones, pero que, con mucho, el mayor beneficio se puede obtener mediante mandatos de vía verde (Harnad, 2010b).

Los mandatos institucionales y de los entes financiadores tienen que ser convergentes y de colaboración (Harnad, 2008) en lugar de divergentes y competitivos. Debe existir un depósito institucional, seguido por una recolección cen- 
tral. Y no un depósito directamente central por mandatos de los financiadores vs. depósito institucional por los mandatos institucionales).

\section{La reforma del copyright vendrá como} una consecuencia, no como condición previa, de la vía verde universal

\section{No exigir demasiado}

Aparte de las dos vías de ofrecer OA (vía verde de auto-archivo y vía dorada de publicación pagada por los autores), hay dos formas o grados: "OA gratuito", que es acceso online sin tener que pagar nada; y "OA libre" que es acceso online gratuito más ciertos derechos de re-uso (que pueden incluir la re-publicación o la mezcla en obras derivadas) (Suber, 2008). Tanto la vía dorada como el OA libre 1) son prematuros y 2) no se pueden imponer, pero la vía verde universal, OA gratuito, preparará el terreno para la vía dorada universal y un cada vez más generalizado OA libre.

El copyright no es un obstáculo para los mandatos de autoarchivo universales; la reforma del copyright vendrá como una consecuencia, no una condición previa, de la vía verde universal. La mayoría de revistas (entre ellas casi todas las de mayor impacto) ${ }^{19}$ ya apoyan el auto-archivo de la versión final del autor, inmediatamente después de la aceptación para la publicación.

Para los artículos de la minoría de revistas que aún no aprueban el auto-archivo inmediato ${ }^{19}$, si el autor quiere respetar el embargo del editor, igualmente puede depositar el documento en un repositorio inmediatamente después de la aceptación, y el acceso al mismo se puede establecer como cerrado en lugar de abierto (Harnad, 2006). Los repositorios tienen la posibilidad de enviar una copia con fines de investigación de los documentos cerrados a cualquier usuario que lo solicite. Se trata de un proceso semi-automático de "envío de eprint bajo demanda" (Sale; Couture; Rodrigues et al., 2010). Esto todavía no es OA, pero es "casi OA", y acelerará el fin de los embargos.

El objetivo principal del OA son las revistas académicas y científicas cuyos artículos han sido evaluados por pares $-2,5$ millones de artículos al año, publicados en 25.000 revistas revisadas por pares, en todas las disciplinas, lenguas y naciones ${ }^{27}$. Cada uno de esos artículos es sin excepción un escrito no comercial, como se describió anteriormente: un regalo del autor, escrito exclusivamente para el bien de la ciencia, su uso, sus aplicaciones y su impacto, y no para obtener ingresos de su venta. Otras formas de contenido digital -libros, libros de texto, artículos de revistas y periódicos, música, vídeo, software- no se han escrito para regalar y en vez de impacto esperan obtener ingresos. Puede suceder que el crecimiento de artículos de investigación evaluados en OA incite a ofrecer más OA también de estas otras formas de contenido, pero es un gran error estratégico tratar los contenidos comerciales de igual forma que los no comerciales, como si fueran la misma clase de cosas, en una vaga noción de "acceso abierto al conocimiento".
Otra forma cada vez más importante de contenidos científicos son los datos básicos o en bruto de la investigación, pero no se puede imponer el acceso abierto inmediato a los mismos porque los investigadores deben disponer de un período razonable de tiempo exclusivo para analizarlos y sacar más conclusiones. Los investigadores también pueden ser animados -aunque no sea obligatorio- a que proporcionen acceso a sus pre-prints (artículos antes de pasar el arbitraje de los pares), pero esto debe seguir siendo una cuestión de elección del autor. Es suficiente que quienes financian la investigación impongan como condición que los datos en que se basa un trabajo de investigación revisado se tengan que hacer públicos una vez que el documento haya sido aceptado para su publicación (con algunas excepciones, por ejemplo si el autor piensa publicar una serie de artículos basados en el análisis de un mismo conjunto de datos).

El tipo de mandato óptimo para el auto-archivo es el modelo de Lieja $^{11}$, que obliga a depositar todos los trabajos aceptados para su publicación en el repositorio institucional como mecanismo único para poder solicitar tanto una evaluación del trabajo en la institución como de la investigación a nivel nacional. Enabling Open Scholarship (EOS) ${ }^{7}$, Open Access Scholarly Information Sourcebook (Oasis) ${ }^{12}$ y Sparc Campus Open Access Policies ${ }^{23}$ ofrecen orientación sobre políticas de cómo diseñar mandatos de OA, dirigida a instituciones y financiadores de investigación.

\section{Es irónico que haya tenido que ser ne- cesario exigir a los investigadores hacer algo que les interesa}

\section{Creación y cuidado del común cognitivo}

Es un hecho histórico irónico -verdadero e indiscutible- que haya tenido que ser necesario exigir a los investigadores que con sus publicaciones no comerciales hicieran un común cognitivo, algo que redundaba abrumadoramente en su propio interés (así como de la humanidad en su conjunto). Los futuros historiadores tendrán que explicar por qué los investigadores no lo hicieron espontáneamente por su propia voluntad una vez que se demostró que era posible, y teniendo los medios (repositorios en OA) para hacerlo. En Southampton también creímos al principio, a mediados de la década de 1990, por supuesto ingenuamente, que pronto lo harían. Biológicamente, y por analogía con el origen del lenguaje, parece como si se tuviera que obligar a la fuerza a las madres a que compartan sus conocimientos (por no hablar de la comida) con su progenie. Quizá poner los resultados de una investigación en OA a disposición de los colegas ofrezca recompensas más directas y palpables aquí y ahora para los autores que hacer la correcta transmisión de los propios genes egoístas a las generaciones posteriores.

Tal vez la razón de que las contribuciones al común cognitivo tengan que ser obligadas está más estrechamente relacionada con el por qué alguien se hace académico o científico (en lugar de comerciante, militar o político). Después de todo, incluso la publicación ha tenido que ser obligada ("publicar o perecer"), de lo contrario una gran cantidad de la investi- 
gación todavía no se habría publicado. Parece como si los investigadores después de haber satisfecho su curiosidad al realizar un estudio luego se quedasen tranquilos poniendo los resultados en un cajón del escritorio.

En la era post-Gutenberg incluso ya no es suficiente la publicación de los resultados de aquellos autores/usuarios cuyas instituciones pueden darse el lujo de tener acceso a ellos mediante una suscripción. Tal vez la mejor forma de resumir la contribución de Southampton a la creación y preservación del común cognitivo es haber ayudado a pasar de la era del mandato "publicar o perecer", -propio de la era Gutenberg-, a la del "auto-archivar para prosperar" -definitorio de la era post-Gutenberg.

\section{Nota}

Este artículo es una traducción realizada por la Redacción de EPI de la comunicación:

Carr, Les; Swan, Alma; Harnad, Stevan. "Creating and curating the cognitive commons: Southampton's contribution", que se presentará en la conferencia Curating the European university. European exposition and public debate, Lovaina, Bélgica, 10-11 de febrero de 2011.

\section{Referencias}

Björk, Bo-Christer; Welling, Patrik; Laakso, Mikael; Majlender, Peter; Hedlund, Turid; Guðnason, Guðni. "Open access to the scientific journal literature: situation 2009". Plos one, 2010, v. 5, n. 6, e11273. DOI: 10.1371/journal. pone.0011273

Brody, Tim; Carr, Les; Gingras, Yves; Hajjem, Chawki; Harnad, Stevan; Swan, Alma. "Incentivizing the open access research web: publication-archiving, data-archiving and scientometrics". CTWatch quarterly, 2007, v. 3, n. 3.

http://eprints.ecs.soton.ac.uk/14418/

Carr, Les; Harnad, Stevan. Keystroke economy: a study of the time and effort involved in self-archiving, 2005.

http://eprints.ecs.soton.ac.uk/10688/

Dror, Itiel E.; Harnad, Stevan. "Offloading cognition onto cognitive technology". In: I. Dror \& S. Harnad (eds.), Cognition distributed: how cognitive technology extends our minds. Amsterdam: John Benjamins, 2009.

http://eprints.ecs.soton.ac.uk/16602/

Gargouri, Yassine; Hajjem, Chawki; Larivière, Vincent; Gingras, Yves; Brody, Tim; Carr, Les; Harnad, Stevan. "Self-selected or mandated, open access increases citation impact for higher quality research". Plos one, 2010, v. 5, n. 10, e13636. http://dx.plos.org/ambra-doi-resolver/10.1371/journal.po ne.0013636

Gargouri, Yassine; Harnad, Stevan. Mandated and unmandated open access: comparing green and gold. Open access archivangelism, 2010.

http://openaccess.eprints.org/index.php?/archives/760guid.html

Harnad, Stevan. "Scholarly skywriting and the prepublication continuum of scientific inquiry". Psychological science, 1990 , v. 1, pp. 342-343.

\section{http://cogprints.org/1581/}

Harnad, Stevan. "Post-Gutenberg galaxy: The fourth revolution in the means of production of knowledge". Public-access computer systems review, 1991, v. 2, n. 1, pp. 39-53. http://cogprints.org/1580/

Harnad, Stevan, 1994.

https://groups.google.com/group/bit.listserv.vpiej-l/browse thread/thread/682843612b4ff4d/62b3bd13407dc1 $d 8 ? h$ l=en\&ie=UTF-8\&q=\%22We+have+heard+many+predict ions+about+the+demise+of+paper+publishing\%22\&pli=1

Harnad, Stevan. "A subversive proposal". In: A. S. Okerson \& J. J. O'Donnell (eds.), Scholarly journals at the crossroads; a subversive proposal for electronic publishing. Washington: Association of Research Libraries, 1995.

http://www.arl.org/sc/subversive/

Harnad, Stevan. For whom the gate tolls? How and why to free the refereed research literature online through author/ institution self-archiving, now. 2001.

http://cogprints.org/1639/

Harnad, Stevan. "The self-archiving initiative". Nature, 410, pp. 1024-1025, 2001.

http://www.nature.com/nature/debates/e-access/Articles/ harnad.html

Harnad, Stevan. Back to the oral tradition through skywriting at the speed of thought, 2003.

http://eprints.ecs.soton.ac.uk/7723/

Harnad, Stevan. The immediate-deposit/optional-access (ID/OA) mandate: rationale and model, 2006, March 13.

http://openaccess.eprints.org/index.php?/archives/71guid.html

Harnad, Stevan. "The green road to open access: a leveraged transition". In: The culture of periodicals from the perspective of the electronic age, pp. 99-105, L'Harmattan, 2007.

Harnad, Stevan. "Waking OA's 'slumbering giant': The university's mandate to mandate open access". New review of information networking, 2008, v. 14, n. 1, pp. 51-68.

http://eprints.ecs.soton.ac.uk/17298/

Harnad, Stevan. How to integrate university and funder open access mandates. Open access archivangelism, 2008, March 2. http://openaccess.eprints.org/index.php?/archives/369guid.htm/2008

Harnad, Stevan. "The PostGutenberg open access journal". In: B. Cope \& A. Phillips (eds.), The future of the academic journal. Cambridge: Chandos Publishing, 2009.

http://eprints.ecs.soton.ac.uk/15617/

Harnad, Stevan. "No-fault peer review charges: the price of selectivity need not be access denied or delayed". D-lib magazine, 2010, v. 16, n. 7-8, Jul.-Aug.

http://www.dlib.org/dlib/july10/harnad/07harnad.html

Harnad, Stevan. "The immediate practical implication of the Houghton report: provide green open access now". Prometheus, 2010b, v. 28, n. 1, pp. 55-59. http://eprints.ecs.soton.ac.uk/18514/

Harnad, Stevan. "Symbol grounding and the origin of language: from show to tell". In: C. Lefebvre \& B. Comry (eds.), 
Origins of language (in press), 2011.

http://eprints.ecs.soton.ac.uk/21438/

Harnad, Stevan; Brody, Tim; Vallières, François; Carr, Les; Hitchcock, Steve; Gingras, Yves; Oppenheim, Charles; Stamerjohanns, Heinrich; Hilf, Eberhard R. "The green and gold roads to open access". Nature web focus, 2004.

http://www.nature.com/nature/focus/accessdebate/21. html

Harnad, Stevan; Carr, Les; Brody, Tim; Oppenheim, Charles. "Mandated online RAE CVs linked to university eprint archives: improving the UK Research Assessment Exercise whilst making it cheaper and easier". Ariadne, 2003, n. 35.

http://www.ariadne.ac.uk/issue35/harnad/

Harnad, Stevan; Carr, Les; Gingras, Yves. "Maximizing research progress through Open Access mandates and metrics". Liinc em revista, 2008, v. 4, n. 2, setembro, Rio de Janeiro, p. 269-273.

http://www.ibict.br/liinc

Hitchcock, Steve. The effect of open access and downloads ('hits') on citation impact: a bibliography of studies, 2011. http://opcit.eprints.org/oacitation-biblio.html

Houghton, John W.; Rasmussen, Bruce; Sheehan, Peter J.; Oppenheim, Charles; Morris, Anne; Creaser, Claire; Greenwood, Helen; Summers, Mark; Gourlay, Adrian. Economic implications of alternative scholarly publishing models: exploring the costs and benefits. London and Bristol: The Joint Information Systems Committee (JISC), 2009.

http://www.jisc.ac.uk/publications/reports/2009/economic publishingmodelsfinalreport.aspx

Lawrence, Steve. "Online or invisible?". Nature, 2001, v. 411, n. 6837, p. 521.

http://www.nature.com/nature/debates/e-access/Articles/ lawrence.html

Poynder, Richard; Harnad, Stevan. From glottogenesis to the category commons. The basement interviews, 2007. http://ia700201.us.archive.org/13/items/The_Basement Interviews/Stevan_Harnad_Interview.pdf

Sale, Arthur H. J. "The acquisition of open access research articles". First monday, 2006a, v. 11, n. 10.

http://eprints.utas.edu.au/388/1/FirstMondayOct06.pdf

Sale, Arthur H. J. "Comparison of IR content policies in Australia". First monday, 2006b, v. 11, n. 4.

http://eprints.utas.edu.au/264/

http://firstmonday.org/issues/issue11_4/sale/

Sale, Arthur H. J.; Couture, Marc; Rodrigues, Eloy; Carr, Les; Harnad, Stevan. "Open access mandates and the 'fair dealing' button". In: R. J. Coombe \& D. Wershler (eds.), Dynamic fair dealing: creating Canadian culture online, 2010.

http://eprints.ecs.soton.ac.uk/18511/

Shadbolt, Nigel; Brody, Tim; Carr, Les; Harnad, Stevan. "The open research web: a preview of the optimal and the inevitable". In: N. Jacobs (ed.), Open access: key strategic, technical and economic aspects. Cambridge: Chandos Publishing, 2006.

Suber, Peter. Green/gold OA and gratis/libre OA. Open ac- cess news, 2008.

http://www.earlham.edu/ peters/fos/2008/08/greengoldoa-and-gratislibre-oa.html

http://openaccess.eprints.org/index.php?/archives/442guid.html

Swan, Alma. Open access self-archiving: An introduction, 2005.

http://eprints.ecs.soton.ac.uk/11006/01/jiscsum.pdf

Swan, Alma. Multiplying mandates. OpenScholarship, 2009.

http://optimalscholarship.blogspot.com/2009/05/ multiplying-mandates.html

\section{Hyperlinks of OA initiatives}

1. American Scientist Open Access Forum http://bit.ly/AmSciForum

2. arXiv

http://arxiv.org/

3. Anonymous FTP

http://bugclub.org/beginners/history/InternetHistory.htm

4. Budapest Open Access Initiative (BOAI)

http://www.soros.org/openaccess/read.shtml

5. Citebase

http://www.citebase.org/

6. CogPrints

http://cogprints.org/

7. Enabling Open Scholarship (EOS)

http://www.openscholarship.org/

8. EPrints

http://www.eprints.org/openaccess/

9. Internet History

http://www.isoc.org/internet/history/brief.shtml

10. IRStats

http://wiki.eprints.org/w/IRStats

11. Liège mandate model http://bit.ly/LiegeOAmandate

12. Open access scholarly information sourcebook (Oasis) http://www.openoasis.org/

13. Open access timeline

http://www.earlham.edu/ peters/fos/timeline.htm

14. Open Archives Initiative (OAI)

http://www.openarchives.org/

15. Open Society Institute (OSI)

http://www.soros.org/

16. OSI Eprints handbook

http://www.eprints.org/documentation/handbook/ universities.php

17. Registry of Open Access Repositories (ROAR) http://roar.eprints.org/

18. Registry of Open Access Materials Archiving Policies (Roarmap)

http://www.eprints.org/openaccess/policysignup/ 
19. Romeo

http://romeo.eprints.org/stats.php

\section{Scoap3}

http://scoap3.org/

\section{Self-archiving $F A Q$}

http://www.eprints.org/openaccess/self-faq/

22. Sigma Xi Scientific Research Society

http://www.sigmaxi.org/

23. Sparc Campus Open Access Policies http://www.arl.org/sparc/advocacy/campus/

24. UK Parliamentary Select Committee on Science and Technology http://www.publications.parliament.uk/pa/cm200304/ cmselect/cmsctech/399/39903.htm

25. US House Appropriations Committee http://bit.ly/UScongressOA

26. UK Research Councils (RCUK)

http://www.rcuk.ac.uk/access/default.htm

27. Ulrichs

http://www.ulrichsweb.com/ulrichsweb/

28. UNIX Timeline

http://www.unix.org/what_is_unix/history_timeline.html

29. World Wide Web History

http://www.w3.org/History.html

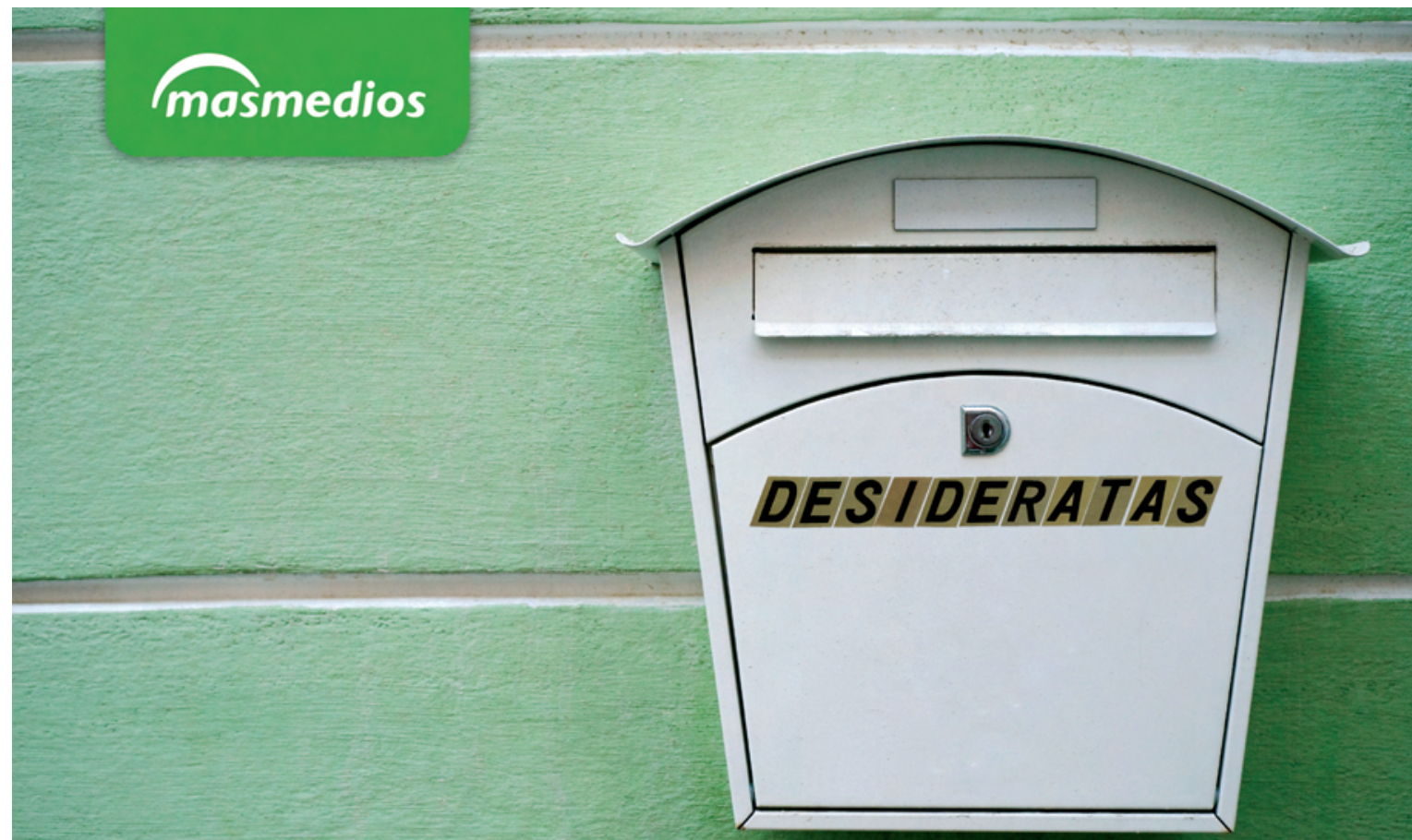

\section{Te entendemos 99}

Sistemas de gestión de bibliotecas Open Source

Interfaces interactivas y OPACs

Repositorios OAl

Gestión documental y de archivos

Digitalización

Outsourcing de servicios documentales

Desarrollo de sitios web / multimedia / e-learning

Comunicación y e-marketing de servicios de información
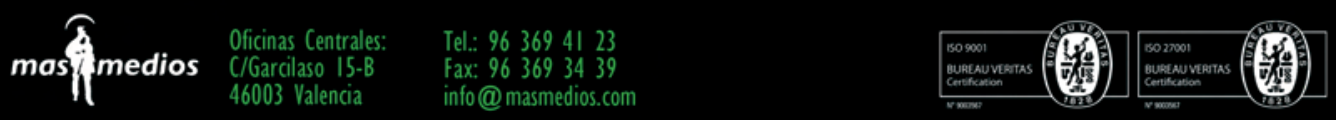